\title{
A MODEL REFERENCE FUZZY ADAPTIVE SLIDING MODE CONTROLLER
}

\author{
Abhijith P R \\ Dept of Electronics and communication \\ RIET, Nagaroor \\ Kerala, India
}

\begin{abstract}
All physical systems are nonlinear in nature. Although nonlinear systems are better they are difficult to control. The Model Reference Adaptive Control (MRAC) that is based on Lyapunov theory is used in conjunction with sliding mode (SM) and fuzzy logic theories and is known as A Model Reference Fuzzy Adaptive Sliding Mode Controller (MRFASMC). In this control scheme, asymptotic tracking of two reference models with different settling times and the different damping conditions is achieved. The outputs do not have oscillation and short settling time. Instead of mathematical modeling of system, fuzzy logic is used for improving transient response of MRAC. The expected advantage of the proposed MRFASMC is to improve the performance specifications in settling time and peak overshoot over the MRAC technique.
\end{abstract}

Keywords - MRAC, Lyapunov, Sliding mode.

\section{INTRODUCTION}

All physical systems in nature have some nonlinearity such as hysteresis, backlash, saturation and dead zone. The various engineering systems such as aircraft, electric vehicles, reactors, inverted pendulum and electric drives are unstable and nonlinear. Even then there are a few advantages to nonlinear systems such as, they can perform better than linear systems, are less costly than linear systems and are usually compact in size when compared with linear systems. There disadvantages include complexity in design and different in control.

The common nonlinear control techniques are backstepping, PID, linear quadratic regulator. They cannot tackle nonlinear systems at all time. Many nonlinear controllers such as Sliding Mode(SM), adaptive, FLC and neural network based controller are used to overcome the nonlinearity problems. Model Reference Adaptive Control (MRAC), is widely used due to its simplicity, less computational efforts and better results. Its aim is to change controller parameters to account for the dynamically varying system parameters or disturbances by comparing output from an ideal plant model called Reference Model (RM) (Duka et al.,2007). The advantage of is that it is capable of tracking the output of RM correctly and suitable RM can be chosen to give desired performance specifications (Shekar et al.,2018). Fuzzy logic systems are simpler since it does not require complicated mathematical computations. Fuzzy helps to improve transient response (Mohideen et al.,2013). Sliding mode control is achieves the asymptotic tracking and guarantee finite time stability. Sliding mode is type of variable structure control. It has continuous as well as discontinuous part. The proposed system has model reference adaptive system for reducing the chattering problem. Usually boundary layer approach is used to reduce the chattering effect. Low pass filter can also filter chattering signal (Jasmheed et al.,2018). The proposed system is evaluated for inverted pendulum. This can be used for vehicle suspension system, electric motor drive.

\section{SYSTEM MODELING}

In this section mathematical model of inverted pendulum is established. Inverted pendulum (IP) is a pendulum system that has its center of mass above its pivot point. It is unstable and without actuator action it will fall over. The system consists of a rigid pole attached to a cart with a hinge, a free joint with one degree of freedom. The cart is constrained to move along a linear horizontal direction when a force is exerted on it. If appropriate forces are applied the pole can be kept in various positions from falling over (Prakash et al.,2016). The cart pole and free body diagram, and the complete block diagram of the IP system for control application are shown in Figure 1.

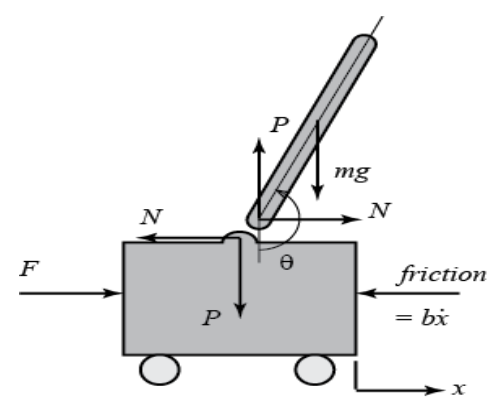

Figure 1. IP Cart Pole Free Body

In Fig 1, F is the applied force acting on the cart. The viscous and static frictional forces in the motion of cart may be considered as disturbance in the IP system. For simplification of open loop transfer, the gain of servomechanism and feedback is considered as unity. The controlled output is angle and let input force be $\mathrm{U}(\mathrm{s})$. The forces on the cart and pendulum in the horizontal direction are given by (3) and (4) (Yadav et al.,2019). 


$$
\begin{gathered}
M \frac{d^{2} x}{d t^{2}}+b \frac{d x}{d t}+N=F \\
M \frac{d^{2} x}{d t^{2}}+m l \frac{d^{2} \theta}{d t^{2}} \cos \theta-m l \theta^{2} \sin \theta=N
\end{gathered}
$$

Using (1) and (2) equation (3) is obtained $(M+m) \frac{d^{2} x}{d t^{2}}+b \frac{d x}{d t}+m l \frac{d^{2} \theta}{d t^{2}} \cos \theta-m l \theta^{2} \sin \theta=F$

Second equation of motion (4) is obtained by summing all force perpendicular to pendulum.

$$
P \sin \theta+N \cos \theta-m g \sin \theta=m l \frac{d^{2} \theta}{d t^{2}}+m \frac{d^{2} x}{d t^{2}} \cos \theta
$$

Assume $\theta=\Pi+\phi$. On solving equation (3), (4) and substituting values given in table 6.1 gives the linearized transfer function of IP as (5).

\begin{tabular}{|ccc|}
\hline Symbol & Description & Values \\
\hline $\mathrm{M}$ & Mass of cart system & $0.6 \mathrm{Kg}$ \\
$\mathrm{m}$ & Mass of the pendulum & $0.25 \mathrm{Kg}^{-1}$ \\
$\mathrm{~b}$ & Friction on cart & $0.11 \mathrm{Nm}^{-1} \mathrm{~S}^{-1}$ \\
$\mathrm{I}$ & Length to pendulum from center of & $0.3 \mathrm{~m}$ \\
$\mathrm{I}$ & mass & $0.0075 \mathrm{Kgm}^{2}$ \\
\hline
\end{tabular}

Table 6.1 Parameters of IP System

$$
\frac{\phi(s)}{U(s)}=\frac{3.78}{s^{2}-31.44}
$$

The linearized IP system (5) is an unstable system because it has two poles on the right half of s plane.

\section{Methodology}

The task is to design a controller which is less affected by parameter variations, external disturbance. Also the inaccuracy in determining reference model should not affect output. The proposed system has mainly 3 sections,
A. Reference section.
B. Fuzzy MRAC.
C. Sliding control.

$$
\frac{\mathrm{C}(s)}{\mathrm{R}(s)}=\frac{w_{\mathrm{n}}{ }^{2}}{s^{2}+2 \zeta w_{\mathrm{n}} s+w_{\mathrm{n}}{ }^{2}}
$$

The Lyapunov approach is used in theoretical analysis in developing the SMC with PID scheme and to ensure that the system is under stable condition. It is used to determine the reaching condition. The control law is based on fuzzy logic and Lyapunov theory for state trajectory to move towards the sliding surface. This is done by choosing a hitting control gain to maintain the derivative of Lyapunov function always negative definite. As chosen Lyapunov the function is negative system will be stable (Yadav et al.,2014,2013)

The Lyapunov function choosen is (7),

$$
\mathrm{V}=\mathrm{s}^{2}
$$

Derivative of Lyapunov is given as (8). This is known as reachability condition (Tianxiang et al.,2016).

$$
v^{*}=s s^{*}
$$

Sliding mode allows robust tracking and ensures finite time stability. Chattering effect introduced by sliding mode can be avoided by fuzzy. Sliding surface chosen is PID sliding surface(9) (Eker ,2006). The term e(t) is the modified error term.

Figure 2. Block Diagram of Proposed MRFASMC 


\section{International Journal of Engineering Applied Sciences and Technology, 2020 Vol. 5, Issue 3, ISSN No. 2455-2143, Pages 549-552 \\ Published Online July 2020 in IJEAST (http://www.ijeast.com)}

Target is to derive $\mathrm{s}=0$. The SMC law can be found by using Lyapunov theory. For stability the first derivative of Lyapunov theory must be negative for all values. The control law is designed to make $v^{\prime}$ negative. It is given as (10). The control law forms the discontinuous part of the sliding control. The invariance or the equivalent function is the sliding surface itself (Azar et al.,2015). The sliding surface is the function of error. First derivative of sliding surface include the input term.

$$
\mathrm{u}_{s}=-\eta \operatorname{sat}\left(\frac{s}{\xi}\right)
$$

Where $\eta$ is the switching control parameter.

Saturation function is defined as;

$$
\operatorname{sat}\left(\frac{s}{\zeta}\right)= \begin{cases}1, & \text { for }\left|\frac{s}{\eta}\right| \leq 1 \\ \operatorname{sgn}\left(\frac{s}{\zeta}\right), \text { for }\left|\frac{s}{\zeta}\right| \geq 1\end{cases}
$$

According to Lyapunov theory, the Lyapunov is positive definite and if the derivative of Lyapunov function is negative definite, then the state trajectory will be driven towards S. Having reached, it will remain sliding on it until the origin $(S=0)$ is reached asymptotically.

Fuzzy controller is used to alter the input error.

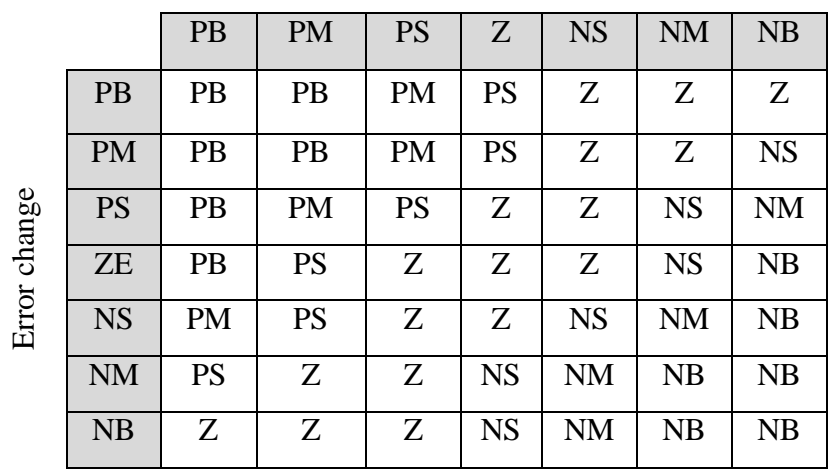

Using fuzzy helps to reduce mathematical complication and introduce artificial intelligence (Abdullah et al.,2008). The fuzzy logic controller is applied to improve the dynamic response, regulation precision and robustness of the system. The fuzzy control subsystem has inputs current error and error derivative. The output of the controlled subsystem follows the output of the reference model. Inputs range is in [-6 6] and output [-1 1]. Seven triangular membership functions are chosen for all variables. The fuzzy rules are shown in table 7.1 (Peng et al.2008).

\section{Error}

\section{Table 1. Fuzzy Control Rule Table}

The output of the fuzzy control subsystem is given by,

$$
\boldsymbol{C}=\mathbf{K} . \mathbf{f} .|\mathbf{e}|
$$

\section{SimUlATION AND RESUlT}

Simulation is done in MATLAB. Set value is a 10 unit step signal. The output obtained for reference model having damping ratio 1 and natural frequency of oscillation as 5 is shown in figure 3. The overshoot is obtained as $0 \%$ and reset time is obtained as $661.3 \mathrm{~ms}$. Settling time is obtained as $1.588 \mathrm{~s}$. Steady state error is zero. The system was able to track reference model faster than PID with MRAC controller. The out obtained is shown in figure 3.

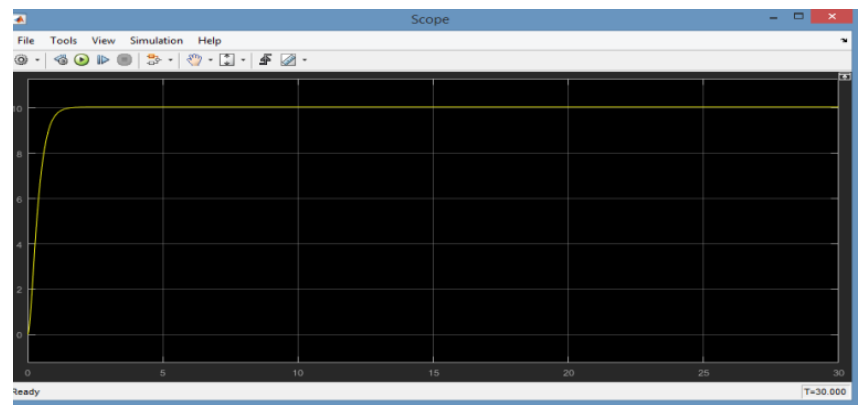

Figure 3. Output of Proposed System

Some disturbance in the form of pulse is provided to the plant along with the input from the controller. The pulse has amplitude of 25 units and period of $0.05 \mathrm{~s}$ and pulse width is $50 \%$ of pulse period. RM 1 is used. The output for disturbance is shown in figure 4.

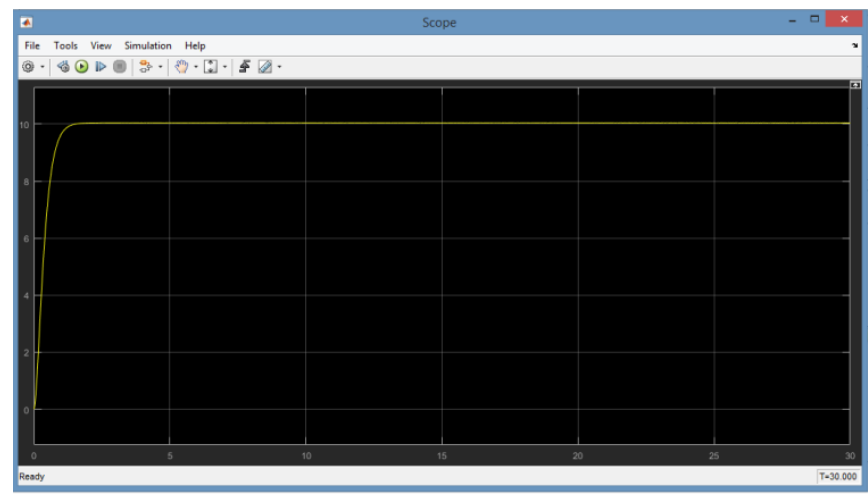

Figure 4. Output of Proposed System with disturbance

\section{CONCLUSION AND FUTURE SCOPE}

The adaptive controls technique is designed and applied to the balance of the highly nonlinear and unstable system. The convergence of the unstable system is guaranteed by Lyapunov theory of stability. Model Reference Fuzzy Adaptive Sliding Mode Controller is designed to make system able to learn and asymptotically track reference model output. MRFASMC settles the response faster as compared to MRAC with least settling time and overshoot. MRFASMC provide better immunity towards parameter variation and disturbances. This may be preferred over MRAC for the precise control in applications such as continuous stirred tank reactor, robot motion control, synchronous generator terminal voltage control used in renewable energy system, electric drives and nonlinear 


\section{International Journal of Engineering Applied Sciences and Technology, 2020 \\ Vol. 5, Issue 3, ISSN No. 2455-2143, Pages 549-552 \\ Published Online July 2020 in IJEAST (http://www.ijeast.com)}

vehicles and may also be applied for robotic tracking, missile tracking control and attitude tracking control of aircraft. In future work fuzzy can be modified to genetic algorithm.

\section{REFERENCES}

1 Peng; Luo; Zhuang.(2008). Model Reference Fuzzy Adaptive PID Control and Its Applications in Typical Industrial Processes, International Conference on Automation and Logistics Qingdao.(pg. 896-901).

2 Abdullah I; Al-Odienat; Al-Lawama.(2008).The Advantages of PID Fuzzy Controllers Over The Conventional Types. American Journal of Applied Sciences. (pg. 653-658).

3 A.K. Yadav; P. Gaur. (2013). Comparative analysis of modern control and AI based control for maintaining constant ambient temperature. World Review of Science, Technology and Sust. Development- Vol. 10 (DOI: 10.1504/WRSTSD.2013.050785).

4 A.K. Yadav; P. Gaur.(2014).AI based adaptive control and design of autopilot system for nonlinear UAV. Indian Academy of Sciences.(pg 765-783).

5 Azar; Serrano. (2015). Adaptive Sliding Mode Control of the Furuta Pendulum. Springer. Advances and Applications in Sliding Mode Control systems. Studies in Computational Intelligence. (DOI 10.1007/978-3-319-11173-5).

6 Tianxiang; Hao.(2016). PID Sliding Mode Controller Design and Application to Active Queue Management. Proceedings of the 35th Chinese Control Conference (pg 27-29).

7 Shekar;sharma.(2018).Review of Model Reference Adaptive Control. International Conference on Information, Communication, Engineering and Technology. (pg 29-31)

8 A.K. Yadav; P. Gaur.(2019). Sliding Mode Based Fuzzy Model Reference Adaptive Control Technique for an Unstable System. The Institution of Engineers India.(DOI: 10.1007/s40031-01900372-5)

9 Isa; Hamza.(2019).Hybrid Fuzzy Control of Nonlinear Inverted Pendulum System. Bayero Journal of Engineering and Technology. (pg 200208).

10 Duka;Oltean; Dulău.(2007). Model Reference Adaptive vs. Learning Control for the Inverted
Pendulum,A Comparative Case Study. CEAI.Vol. 9 (pg 67-75)

11 Mohideen;Valarmathi.(2013).Fuzzy Modified Model Reference Adaptive Controller for Improved Transient Response. International Conference on Power, Energy and Control. (pg. 454 -457).

12 Eker. (2006). Sliding Mode Control with PID Sliding Surface and Experimental Application to Electromechanical Plant. ISA Transactions. (pg. 109-118)

13 Kutlu;Atan.(2019).Intuitionistic Fuzzy Adaptive Sliding Mode Control of Nonlinear Systems.springer. (DOI: 10.1007.s00500-01904286-8).

14 Prakash;KrishnaRoy;Biswas.(2016).Design, Implementation and Comparison of Different Controllers for a Rotary Inverted Pendulum. IEEE International Conference on Power Electronics, Intelligent Control and Energy Systems.(DOI: 978$1-4673-8587-9 / 16)$

15 Jasmheed;Abbasi.(2018).Chattering Reduction by Using Propotional Derivative Sliding Surface in Sliding Mode Control. International Conference on Information and Communication Technology Robotics. (DOI 10:1109/ICTROBOT.2018.8549911) 